\title{
Direct Evidence for a Dynamical Ground State in the Highly Frustrated $\mathrm{Tb}_{2} \mathrm{Sn}_{2} \mathrm{O}_{7}$ Pyrochlore
}

\author{
F. Bert, ${ }^{1}$ P. Mendels, ${ }^{1}$ A. Olariu, ${ }^{1}$ N. Blanchard, ${ }^{1}$ G. Collin, ${ }^{2}$ A. Amato, ${ }^{3}$ C. Baines,${ }^{3}$ and A. D. Hillier ${ }^{4}$ \\ ${ }^{1}$ Laboratoire de Physique des Solides, UMR 8502, Université Paris-Sud, 91405 Orsay, France \\ ${ }^{2}$ Laboratoire Léon Brillouin, DSM, CEA Saclay, 91191 Gif-sur-Yvette Cedex, France \\ ${ }^{3}$ Paul Scherrer Institut, Laboratory for Muon Spin Spectroscopy, CH-5232, Villigen PSI, Switzerland \\ ${ }^{4}$ ISIS Facility, CLRC Rutherford Appleton Laboratory Chilton, Didcot, Oxon OX11 OQX, United Kingdom
}

(Received 7 March 2006; published 15 September 2006)

\begin{abstract}
$\mu \mathrm{SR}$ experiments have been performed on a powder sample of the "ordered spin ice" $\mathrm{Tb}_{2} \mathrm{Sn}_{2} \mathrm{O}_{7}$ pyrochlore. At base temperature $(T=35 \mathrm{mK})$, the muon relaxation is found to be of dynamical nature, which demonstrates that strong fluctuations persist below the ferromagnetic transition $\left(T_{C}=0.87 \mathrm{~K}\right)$. Hints of long-range ordering appear as oscillations of the muon polarization when an external field is applied and also as a hysteretic behavior below $T_{C}$. We propose that dynamics results from fluctuations of clusters of correlated spins with the ordered spin ice structure.
\end{abstract}

PACS numbers: 75.40.Gb, 75.10.Nr, 76.75.+i

In the field of highly frustrated magnetism, rare-earth (R) titanate pyrochlores $R_{2} \mathrm{Ti}_{2} \mathrm{O}_{7}$ have attracted much attention in the past decade. As a result of large spin values of the $R$ atoms and usually small exchange coupling, spin anisotropy, dipolar and exchange interactions compete. The delicate balance of these energy scales, which is specific to each particular pyrochlore compound, stabilizes original magnetic phases from spin ice $(R=\mathrm{Ho}$, Dy) to a unique and still debated collective paramagnetism in $\mathrm{Tb}_{2} \mathrm{Ti}_{2} \mathrm{O}_{7}$ [1]. External constraints such as magnetic field or pressure can easily destabilize this balance and drive the system into complex phase diagrams [2,3]. In the same trend of idea, special attention was recently devoted to the closely related stannate pyrochlore $R_{2} \mathrm{Sn}_{2} \mathrm{O}_{7}$, where lattice expansion as well as modification of the $R$ oxygen environment can also result in drastic changes of the balance of the interactions and eventually in novel exotic ground states [4]. Indeed, while the titanate and stannate Tb pyrochlores exhibit similar antiferromagnetic correlations at high temperature, the Ti compound remains disordered and dynamical down to $70 \mathrm{mK}$, whereas the stannate counterpart undergoes a "ferromagnetic" transition at $0.87 \mathrm{~K}$. It was recently proposed from neutron experiments [5] that the stannate compound freezes in an original uniform $(\mathbf{q}=0)$ spin ice structure where the four spins located at the vertices of each tetrahedron obey the "two in, two out" ice rules [6]. The unexpected ferromagnetism results then from the alignment of the vector sums of the four spins on each tetrahedron. Interestingly, the $\mathrm{Tb}^{3+}$ frozen moment deduced from a nuclear Schottky anomaly in heat capacity measurements [5] was found to be nearly twice smaller than the one extracted from neutron scattering. Despite the static neutron picture, this suggested the existence of slow fluctuations, out of the neutron time window.

The coexistence of fluctuations, the fingerprint of frustration, and glassy behavior [7-10] appears as a widespread and poorly understood feature of many frustrated systems. Even more surprising is the recently reported coexistence of spin dynamics and long-range ordering, well below the transition temperature of some pyrochlore compounds $(R=\mathrm{Gd}, \mathrm{Er})$ [11-13]. Using the muon spin relaxation technique $(\mu \mathrm{SR})$, we directly detect large spin fluctuations in the $\mathrm{Tb}_{2} \mathrm{Sn}_{2} \mathrm{O}_{7}$ compound which we attribute to fast reorientations of spin clusters among the sixfold degenerate ground state of the ordered spin ice structure.

With a large gyromagnetic ratio $\gamma_{\mu}=2 \pi \times$ $13.55 \mathrm{kHz} / \mathrm{G}$ and a weak coupling to its magnetic environment, the muon is a very sensitive probe of magnetism. The accessible time window usually falls in between that of NMR and neutron experiments. Positive muon $\mu^{+}$is therefore an invaluable local probe of spin fluctuations in highly frustrated magnets. As a noticeable example, $\mu$ SR gave the first direct evidence of a fluctuating ground state in the archetypal kagomé bilayers $\mathrm{SrCr}_{9 p} \mathrm{Ga}_{12-9 p} \mathrm{O}_{19}$ [7]. In a $\mu \mathrm{SR}$ experiment, the asymmetry of the $\mu^{+}$decay between forward and backward positron detectors is recorded as a function of the muon lifetime $t$ in the sample [14]. The asymmetry $a(t)$ is proportional to the muon spin polarization, which equals 1 at $t=0$ since the muon beam is $100 \%$ spin polarized.

$\mu$ SR measurements on powder samples of $\mathrm{Tb}_{2} \mathrm{Sn}_{2} \mathrm{O}_{7}$ in zero (ZF) and longitudinal (LF) applied field-with respect to the muon initial polarization-were performed at the ISIS and PSI (Paul Scherrer Institut) facilities. In the following figures, in order to allow direct comparison of spectra obtained with different experimental setups, we plot the quantity $P(t)=\left(a(t)-a_{\mathrm{bkg}}\right) /\left(a(0)-a_{\mathrm{bkg}}\right)$, where $a_{\mathrm{bkg}}$ stands for the background signal arising from muons which missed the sample. The samples were synthesized by standard solid state reaction. X-ray diffraction and SQUID magnetization measurements are in agreement with previously published data $[4,5]$.

The time dependence of the muon polarization has been recorded from room temperature down to $30 \mathrm{mK}$ in a small longitudinal field $H_{\mathrm{LF}}=50 \mathrm{G}$. It can be fitted to a 
stretched exponential function

$$
P(t)=e^{-(\lambda(T) t)^{\alpha}}+B,
$$

$\alpha$ being close to 1 in the whole temperature range. The $T$-independent $B \simeq 10 \%$ term stands for the restored polarization of the muons which experience internal fields, typically of nuclear origin, much smaller than $H_{\mathrm{LF}}$. The muon relaxation rate $\lambda(T)$ is presented in Fig. 1. In the fast fluctuation limit and for a single time relaxation process, $\lambda$ is related to the electronic spin fluctuations rate $\nu$ by [15]

$$
\lambda=\frac{2 \gamma_{\mu}^{2} H_{\mu}^{2} \nu}{\nu^{2}+\gamma_{\mu}^{2} H_{\mathrm{LF}}^{2}},
$$

where $H_{\mu}$ is the magnitude of the fluctuating field experienced by the muon. For zero or small external field, we thus get $\lambda \propto 1 / \nu$. While the muon relaxation rate hardly depends on temperature at high temperatures, as expected for paramagnetic fluctuations, it steeply increases between 10 and $\simeq 1 \mathrm{~K}$, reflecting a strong slowing down of spin fluctuations at the approach of the ferromagnetic transition at $T_{C}=0.87 \mathrm{~K}$. Below $T_{C}$ and down to the lowest temperature of the experiment $T=30 \mathrm{mK}$, the muon relaxation rate saturates at a constant value. As depicted in the inset in Fig. 1, there is surprisingly no qualitative change in the shape of $P(t)$ above and below $T_{C}$. In particular, the usual signs of (i) a static ground state, namely, the powder average long time tail $P(t \rightarrow \infty, T \rightarrow 0)=1 / 3$, and (ii) long-range ordering, namely, oscillations of the polarization due to a well defined internal field, are not observed.

In order to determine the nature, either static or dynamical, of the $\mu^{+}$relaxation at base $T$, we studied the magnetic field dependence of $P\left(H_{\mathrm{LF}}, t\right)$. Were the relaxation at $T=$ $35 \mathrm{mK}$ due to a static local field $H_{\mu}^{S}$ at the muon site or, in a more disordered scenario, a distribution of width $H_{\mu}^{S}$ of such static fields, $H_{\mu}^{S}$ would approximatively be given by $\lambda / \gamma_{\mu}$. The full muon polarization $P\left(H_{\mathrm{LF}}, t\right)$ should then be restored for $H_{\mathrm{LF}} / H_{\mu}^{S} \gtrsim 5$ [14]. At $35 \mathrm{mK}, \lambda / \gamma_{\mu} \simeq 35 \mathrm{G}$,

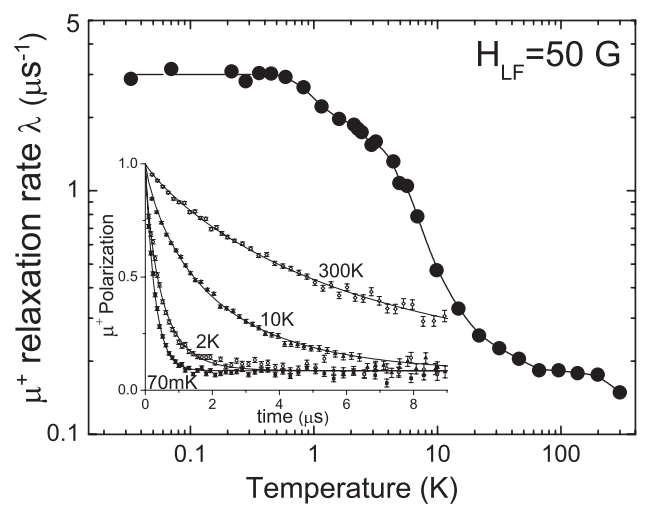

FIG. 1. Variation with temperature of the muon relaxation rate $\lambda$ in a small $50 \mathrm{G}$ longitudinal field on a log- log scale. Inset: Muon polarization versus time at some selected temperatures, also in $50 \mathrm{G}$. and, as shown in Fig. 2, the relaxation is still strong under a $2500 \mathrm{G}$ applied field. Therefore, we can safely conclude that the relaxation of the muon polarization is of a dynamical nature. For a dynamical relaxation, which involves $T_{1}$ processes, much higher fields are needed to restore the initial polarization. The field dependence is then given theoretically by Eq. (2). Experimentally, in $\mathrm{Tb}_{2} \mathrm{Sn}_{2} \mathrm{O}_{7}$, it appears to be rather complicated, and we discuss it in more detail in the following. However, if one restricts to high field and long time relaxation $(t \geq 0.1 \mu \mathrm{s})$ as in Fig. 2, $\lambda\left(H_{\mathrm{LF}}\right)$ nicely obeys Eq. (2) and we can extract $H_{\mu} \simeq$ $200 \mathrm{G}$ and $\nu \simeq 0.2 \mathrm{GHz}$ in a straightforward manner. This fluctuation rate is below the accessible neutron diffraction time window (typically in the gigahertz to terahertz range). Note that the extracted field $H_{\mu}$ is surprisingly small given the high spin value of $\mathrm{Tb}$ ions. It may point out more complicated dynamical processes than described by Eq. (2). At higher temperatures, the $T_{1}=1 / \lambda$ processes progressively evolve to an unexpected linear dependence with $H_{\mathrm{LF}}$ as shown in the inset in Fig. 2. Such a linear field dependence of $1 / \lambda$ was formerly observed in $\mathrm{Tb}_{2} \mathrm{Ti}_{2} \mathrm{O}_{7}[16,17]$ and suggests similar dynamics in both compounds. In the case of $\mathrm{Tb}_{2} \mathrm{Ti}_{2} \mathrm{O}_{7}$, this behavior was tentatively described by a nonconventional power law decay of the spin autocorrelation function.

We now address the second feature of the ZF relaxation shape below $T_{C}$, namely, the absence of spontaneous oscillations. From the magnetic neutron Bragg peaks, a finite correlation length $l_{C} \simeq 18 \mathrm{~nm}$ was extracted [3]. This is quite large compared to the length scale set by the dipolar coupling of the muon, which should therefore sense a well defined internal field. An oscillation of the asymmetry should then be detected, even in the presence of magnontype excitations or paramagnetic centers. Indeed, these sources of dynamics can yield a reduction of the order

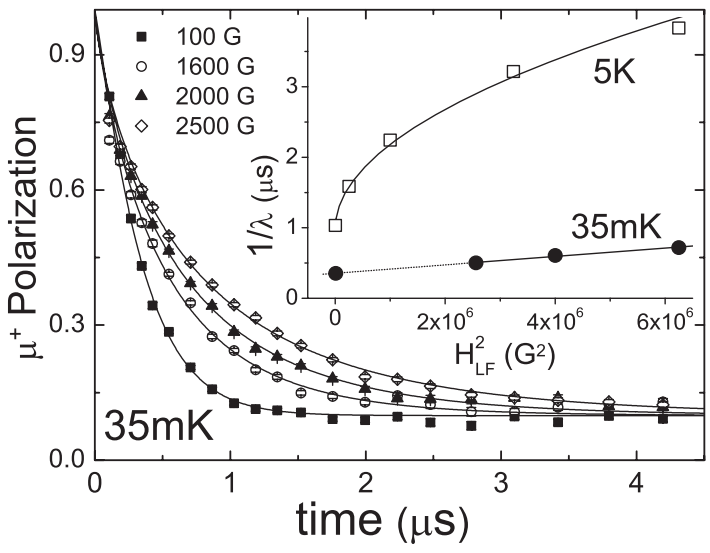

FIG. 2. Field dependence of the muon polarization relaxation at $35 \mathrm{mK}$ for large applied fields (ISIS data). Inset: Relaxation rates deduced from fits of the polarization with Eq. (1) (solid lines in main figure) plotted against $H_{\mathrm{LF}}^{2}$ at $35 \mathrm{mK}$ and for comparison at $5 \mathrm{~K}$ well above $T_{C}$. The lines are linear fits of $1 / \lambda$ versus $H_{\mathrm{LF}}^{2}$ at $35 \mathrm{mK}$ and $H_{\mathrm{LF}}$ at $5 \mathrm{~K}$. 
parameter and/or a damping of the oscillations as well as the relaxation of the long time $1 / 3$ rd tail but do not wipe out the oscillations. The absence of oscillations is thus a meaningful and unusual feature which reveals novel and more drastic fluctuations. We first notice that the average field at the muon site has to be zero; i.e., there should be no underlying static order. If the field at the muon site is $H_{\mu}$ at a given time, it has to fully reverse to $-H_{\mu}$ on the time scale $1 / \nu$. For fast fluctuations $\nu \gtrsim \gamma_{\mu} H_{\mu}$, one indeed gets an exponential-like decay of $P(t)$ (see, for instance, a comprehensive calculation in Ref. [17]). Second, we recall that the ordered spin ice state is sixfold degenerate. One can indeed choose the two in and two out spins out of the four on one tetrahedron or, equivalently, the direction of the resulting moment among one of the six (100)-type directions. If one state yields a field $H_{\mu}$ at the muon site then, the opposite state obtained by reversing all the spins is also in the ground state and yields a field $-H_{\mu}$ at the muon site, while the spin-spin correlations are preserved. We thus propose that the whole spin cluster which surrounds the muon probe and creates $H_{\mu}$ fluctuates in between the six configurations of the ordered spin ice state. The small fluctuation rate $\nu \simeq 0.2 \mathrm{GHz}$ that we find supports cluster fluctuations rather than single spin ones. In order to comply with the relatively large correlation length $l_{C}$, measured on a much faster time scale by neutron experiments, the excitations cannot be localized. They should rather extend over domains of typical size $l_{C}$, either in a coherent fluctuation state, which would then involve an impressive large number of spins, or more probably by a domain wall motion mechanism. To summarize, in the proposed scenario, the ferromagnetic transition corresponds to the freezing of the spin correlations on a large but finite length scale $l_{C}$ which defines the extent of "longrange ordering." This order is dynamical in the sense that

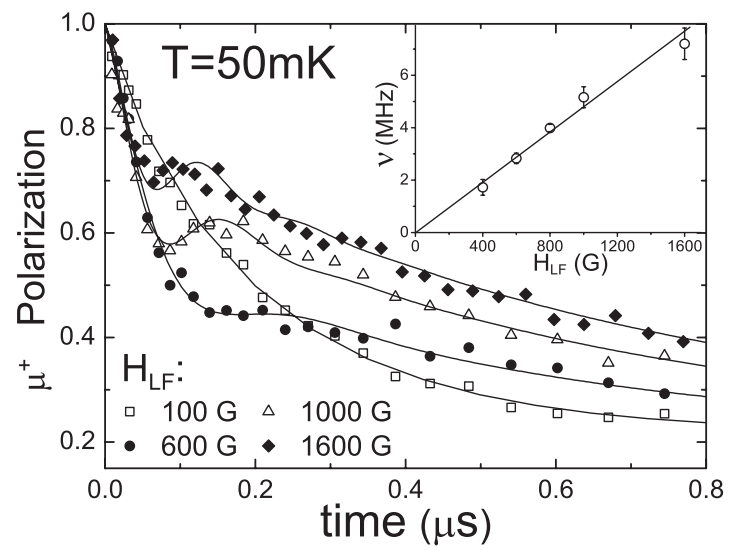

FIG. 3. Field dependence of the polarization relaxation for not too large fields (PSI data). Solid lines are phenomenological fits to the sum of a damped cosine and a stretched exponential function used to track the early time oscillation frequency $\nu$, which is plotted in the inset. the ordered clusters undergo global rotations while the spin-spin correlations are preserved.

In our $\mu$ SR study, the long-range-ordering ground state of $\mathrm{Tb}_{2} \mathrm{Sn}_{2} \mathrm{O}_{7}$ is only clearly evidenced in the detailed analysis of magnetic field effects on the $\mu^{+}$relaxation. As shown in Fig. 3, for intermediate values of the applied longitudinal fields $H_{\mathrm{LF}}$, smaller than the ones used in Fig. 2, the relaxation is no more exponential but exhibits a strongly damped oscillation at early times. The frequency of this oscillation is plotted in the inset in Fig. 3. It scales linearly with the applied field according to $\nu=$ $A \gamma_{\mu} H_{\mathrm{LF}} / 2 \pi$, with $A \simeq 0.37$. Field induced oscillations are commonly observed in disordered magnets around their transition temperature $[15,18]$; however, the frequency is then precisely $\gamma_{\mu} H_{\mathrm{LF}} / 2 \pi . A \ll 1$ rules out such an explanation here. The oscillations are more likely a consequence of long-range ordering in agreement with the proposed cluster scenario. $A$ should then be associated with a magnetic susceptibility resulting from progressive spin canting along the applied field. The effect of the external field is to break the high symmetry of the ground state. Hence, the mean vector field at the muon site is no more zero and oscillations are observed.

After applying increasing longitudinal fields at base temperature as described above, we interestingly did not recover the same polarization decay in zero field. Namely, the relaxation rate was slightly higher after the field experiment than before, although the polarization versus time plots are qualitatively similar (see inset in Fig. 4). Relatively strong applied fields $\left(H_{\mathrm{LF}} \gtrsim 800 \mathrm{G}\right)$ are likely to drive the system in a different spin configuration, resulting in a higher field $H_{\mu}+\Delta H_{\mu}$ at the muon site. Considering the small magnitude of $\Delta H_{\mu}$ [a fit with Eq. (1) gives $\Delta H_{\mu} / H_{\mu} \simeq 15 \%$ at $35 \mathrm{mK}$, one can assume that only minor modifications of the overall ordered spin ice structure subsist after application and removal of the

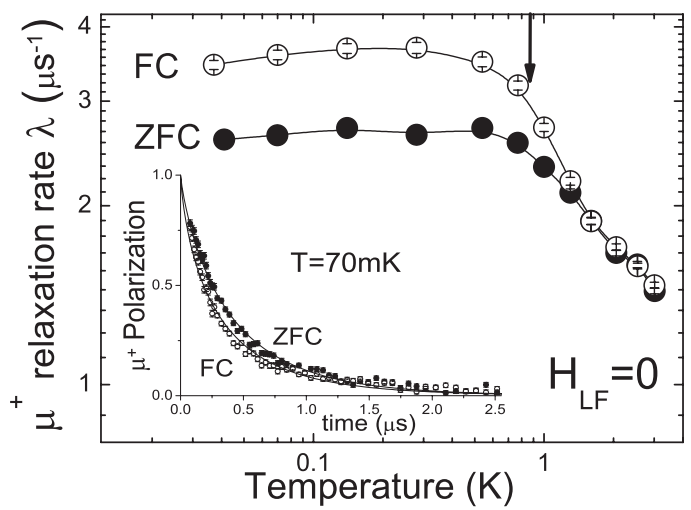

FIG. 4. Muon relaxation rate $\lambda$ deduced from fits with Eq. (1) of the zero field polarization versus time data measured after cooling down the sample below $2 \mathrm{~K}$ in zero external field (ZFC) or with $H_{\mathrm{LF}}=800 \mathrm{G}$ (FC). The inset shows the raw zero field polarization at base temperature for the two cooling procedures. 
magnetic field. We checked that the effect of an external magnetic field as presented in Figs. 2 and 3 hardly depends on the initial zero field state. Thus, despite a slight change in the spin configurations, the ground state remains dynamical. Such an hysteresis effect is a signature of frozen spin correlations as we expect below $T_{C}$. Field history dependences have, for instance, been reported in the spin ice $\mathrm{Ho}_{2} \mathrm{Ti}_{2} \mathrm{O}_{7}$ compound in neutron [19] and $\mu \mathrm{SR}$ [20] experiments. We further investigated this effect by measuring the muon relaxation rate as a function of temperature from $70 \mathrm{mK}$ up to $3 \mathrm{~K}$ in zero external field after (i) cooling down the sample from $3 \mathrm{~K}$ in zero external field (ZFC) and (ii) cooling down the sample with $H_{\mathrm{LF}}=800 \mathrm{G}$ and then removing the external field at base temperature (FC). The results are plotted in Fig. 4. Interestingly, the hysteresis starts at a somewhat higher temperature $(T \simeq$ $1.3 \mathrm{~K}$ ) than the actual transition temperature defined by the peak in the heat capacity measurements at $T_{C}=0.87 \mathrm{~K}$. This perfectly agrees with neutron measurements where the magnitude of the frozen $\mathrm{Tb}$ moment was shown to vanish only above $1.3 \mathrm{~K}$. These results point at a rather broad transition regime.

To summarize, we have evidenced that a dynamical regime survives in $\mathrm{Tb}_{2} \mathrm{Sn}_{2} \mathrm{O}_{7}$ far below its ferromagnetic transition. This compound therefore joins the recently exhibited class of pyrochlores where fluctuations and long-range ordering are simultaneously observed, namely, $\mathrm{Gd}_{2} \mathrm{Ti}_{2} \mathrm{O}_{7}, \mathrm{Gd}_{2} \mathrm{Sn}_{2} \mathrm{O}_{7}$ and $\mathrm{Er}_{2} \mathrm{Ti}_{2} \mathrm{O}_{7}$. Our results for $\mathrm{Tb}_{2} \mathrm{Sn}_{2} \mathrm{O}_{7}$ are, however, markedly different from the $\mathrm{Gd}_{2} \mathrm{Ti}_{2} \mathrm{O}_{7}$ case, where fluctuations were observed in addition to oscillations of the muon relaxation. Here the exponential decay of the muon polarization cannot be ascribed to fluctuations around an overall long-range ordering but results from full fluctuations of the local field. In order to get a consistent picture with neutron results [3], we propose that whole clusters of well ordered spins fluctuate in between the six degenerate configurations allowed in the "ordered spin ice" structure. Application of an external longitudinal field breaks the symmetry of the configuration subspace and oscillations can be detected. Frozen correlations are also evidenced by a field history dependence of the zero field muon relaxation below $\simeq T_{C}$.

The model we propose to describe the low $T$ dynamics demands a unique configuration space with small energy barriers in between the sixfold degenerate configurations of the ground state in order to allow for low energy collective excitations. This is rather counterintuitive with respect to the Ising-type anisotropy which stabilizes the local spin ice order. Contrary to spin ice pyrochlores, the ordered spin ice structure may not be dominantly driven by single ion anisotropy, and the latter may not be too strong in $\mathrm{Tb}_{2} \mathrm{Sn}_{2} \mathrm{O}_{7}$ as proposed in the parent compound $\mathrm{Tb}_{2} \mathrm{Ti}_{2} \mathrm{O}_{7}$ [21]. It is also noticeable that, despite the ferromagnetic transition of $\mathrm{Tb}_{2} \mathrm{Sn}_{2} \mathrm{O}_{7}$, the muon relaxation is strikingly similar to a disordered $\mathrm{Tb}_{2} \mathrm{Ti}_{2} \mathrm{O}_{7}$ magnet. One may assume that the dynamics observed here is not different in nature from the collective excitations of $\mathrm{Tb}_{2} \mathrm{Ti}_{2} \mathrm{O}_{7}$ $[1,16]$. Understanding the exotic dynamical ground state of $\mathrm{Tb}_{2} \mathrm{Sn}_{2} \mathrm{O}_{7}$ where correlations are well defined may, in turn, bring new insight to the puzzling and more complex case of the Ti counterpart.

The authors thank R. Moessner for fruitful discussions. This research project has been supported by the European Commission under Framework Programme 6 through the Key Action: Strengthening the European Research Area, Research Infrastructures, Contract No. RII3-CT-2003505925 .

[1] J. S. Gardner et al., Phys. Rev. Lett. 82, 1012 (1999).

[2] O. Pentrenko, M. Lees, G. Balakrishnan, and D. M. Paul, Phys. Rev. B 70, 012402 (2004).

[3] I. Mirebeau and I. Goncharenko, Phys. Rev. Lett. 93, 187204 (2004).

[4] K. Matsuhira et al., J. Phys. Soc. Jpn. 71, 1576 (2002).

[5] I. Mirebeau et al., Phys. Rev. Lett. 94, 246402 (2005).

[6] J. Snyder, J. S. Slusky, R. J. Cava, and P. Schiffer, Nature (London) 413, 48 (2001); S. T. Bramwell and M.J.P. Gingras, Science 294, 1495 (2001).

[7] Y. Uemura et al., Phys. Rev. Lett. 73, 3306 (1994).

[8] S. Dunsiger et al., Phys. Rev. B 54, 9019 (1996).

[9] F. Bert et al., Phys. Rev. Lett. 95, 087203 (2005).

[10] D. Bono et al., Phys. Rev. Lett. 93, 187201 (2004).

[11] E. Bertin et al., Eur. Phys. J. B 27, 347 (2002).

[12] A. Yaouanc et al., Phys. Rev. Lett. 95, 047203 (2005).

[13] J. Lago et al., J. Phys. Condens. Matter 17, 979 (2005).

[14] For an introduction review, see, e.g., S. J. Blundell, Contemp. Phys. 40, 175 (1999).

[15] R. Hayano et al., Phys. Rev. B 20, 850 (1979).

[16] A. Keren et al., Phys. Rev. Lett. 92, 107204 (2004).

[17] A. Keren, J. Phys. Condens. Matter 16, S4603 (2004).

[18] A. Keren, F. Gulener, I. Campbell, G. Bazalitsky, and A. Amato, Phys. Rev. Lett. 89, 107201 (2002).

[19] M. J. Harris, S. T. Bramwell, D. F. McMorrow, T. Zeiske, and K. W. Godfrey, Phys. Rev. Lett. 79, 2554 (1997).

[20] M. J. Harris, S. T. Bramwell, T. Zeiske, D. F. McMorrow, and P. J. C. King, J. Magn. Magn. Mater. 177, 757 (1998).

[21] Y. J. Kao, M. Enjalran, A. Del Maestro, H. R. Molavian, and M. J. P. Gingras, Phys. Rev. B 68, 172407 (2003). 